\title{
FACTORIZATION OF POSITIVE CONES OF ORDER $n$ OF VON NEUMANN ALGEBRAS
}

\author{
YASUHIDE MIURA \\ (Communicated by Palle E. T. Jorgenson) \\ Dedicated to Professor Jun Tomiyama on his 60th birthday
}

\begin{abstract}
In this paper we shall consider the factorization of positive cones of order $n$ of a von Neumann algebra. Namely, we shall show the existence of a *-subalgebra inducing the positive cone of order $n$ of a von Neumann algebra.
\end{abstract}

Let $M$ be a von Neumann algebra on a Hilbert space $H$. It is known that a positive cone $M_{n}(M)^{+}\left(=\left(M \otimes M_{n}\right)^{+}\right)$, where $M_{n}$ denotes an algebra of all $n \times n$ matrices, coincides with the convex hull of all elements $\left[x_{i}^{*} x_{j}\right]$ for $x_{i} \in M$. We shall find a $*$-subalgebra $N$ of $M$ such that a positive cone of order $n$ is generated by all elements $\left[a_{i}^{*} c^{*} c a_{j}\right]$ for $a_{i} \in N, c \in M$. We may then say that the cone is factorized by $N$. The purpose of this paper is to consider when a positive cone of order $n$ can be factorized or not. The factorization of a self-dual cone in the Hilbert space associated with a standard form of a von Neumann algebra was already shown in [4]. We shall use the books of Pedersen [5] and Takesaki [8] as references of concepts and results of operator algebras.

We begin with the following definition, in which we consider two convex cones of order $n$.

Definition 1. Let $M$ be a von Neumann algebra on a Hilbert space $H$ and $N$ a subspace of $M$. For a natural number $n$ we put

$$
\begin{gathered}
C_{n}(N)=\overline{\operatorname{co}}^{\mathrm{s}}\left\{\left[a_{i}^{*} c^{*} c a_{j}\right] \in M_{n}(M) \mid a_{i} \in N, c \in M\right\}, \\
D_{n}(N)=\left\{\left[x_{i, j}\right] \in M_{n}(M) \mid \sum_{i, j=1}^{n} a_{i}^{*} x_{i, j} a_{j} \geq 0 \text { for every } a_{1}, \ldots, a_{n} \in N\right\},
\end{gathered}
$$

where $\overline{\mathrm{co}}^{\mathrm{s}}$ denotes the strongly closed convex hull.

It is clear that $C_{n}(N) \subset M_{n}(M)^{+} \subset D_{n}(N)$. In particular, if $N=M$, these cones are equal.

Received by the editors June 29, 1992.

1991 Mathematics Subject Classification. Primary 46L10.

Key words and phrases. Positive cone, positive map, completely positive map, injective factor, von Neumann algebra of type I. 
We now show the condition for $N$ that $D_{n}(N)$ coincides with the positive cone of order $n$ of $M$.

Proposition 2. Let $M$ be a von Neumann algebra on a Hilbert space $H$ and $\pi$ a faithful *-representation of $M$ on a Hilbert space $K$. If $N$ is a subspace of $M$ such that $\pi(N)$ has a cyclic vector (i.e., $\pi(N) \xi$ is dense in $K$ for some $\xi \in K)$, then $M_{n}(M)^{+}=D_{n}(N), n \in \mathbb{N}$.

Proof. Choose the element $\xi$ in $K$ as above. If $\left[x_{i, j}\right]$ belongs to $D_{n}(N)$, then

$$
\begin{aligned}
& \left(\left[\begin{array}{ccc}
\pi\left(x_{1,1}\right) & \cdots & \pi\left(x_{1, n}\right) \\
\vdots & & \vdots \\
\pi\left(x_{n, 1}\right) & \cdots & \pi\left(x_{n, n}\right)
\end{array}\right]\left[\begin{array}{c}
\pi\left(a_{1}\right) \xi \\
\vdots \\
\pi\left(a_{n}\right) \xi
\end{array}\right],\left[\begin{array}{c}
\pi\left(a_{1}\right) \xi \\
\vdots \\
\pi\left(a_{n}\right) \xi
\end{array}\right]\right) \\
& =\left(\sum_{i, j=1}^{n} \pi\left(x_{i, j}\right) \pi\left(a_{j}\right) \xi, \pi\left(a_{i}\right) \xi\right) \\
& =\left(\pi\left(\sum_{i, j=1}^{n} a_{i}^{*} x_{i, j} a_{j}\right) \xi, \xi\right) \geq 0,
\end{aligned}
$$

for every $a_{i} \in N$. Therefore, $\left[\pi\left(x_{i, j}\right)\right] \geq 0$, so $\left[x_{i, j}\right] \geq 0$. It follows that [ $\left.x_{i, j}\right]$ belongs to $M_{n}(M)^{+}$. This completes the proof.

We immediately obtain the following examples from the above proposition.

Example 3. Suppose that

$$
M=M_{k(1)} \oplus \cdots \oplus M_{k(m)}, \quad k(1) \leq \cdots \leq k(m) .
$$

Put

$$
A=\left\{\left[\begin{array}{llll}
\lambda_{1} & & & 0 \\
& \lambda_{2} & & \\
& & \ddots & \\
0 & & & \lambda_{k(1)}
\end{array}\right] \oplus \cdots \oplus\left[\begin{array}{llll}
\lambda_{1} & & & 0 \\
& \lambda_{2} & & \\
& & \ddots & \\
0 & & & \lambda_{k(m)}
\end{array}\right] .\right.
$$

Since the subalgebra of all diagonal matrices of $M_{k(i)}$ has a cyclic vector in $H_{k(i)}$ where $H_{k}$ means a $k$-dimensional Hilbert space, $M_{n}(M)^{+}$coincides with $D_{n}(A)$ for $n \in \mathbb{N}$ by the above proposition (cf. [4, Lemma 2.1]).

Another example is given by $M_{n}\left(M_{k m}\right)^{+}=D_{n}\left(M_{k} \otimes I_{m}\right), n \in \mathbb{N}$, with $k \geq m$.

Lemma 4. Let $M$ be a $\sigma$-finite, finite von Neumann algebra on a Hilbert space $H$ and $N$ a von Neumann subalgebra of $M$. Then, for each natural number $n$, the following conditions are equivalent:

(i) $M_{n}(M)^{+}=C_{n}(N)$.

(ii) $M_{n}(M)^{+}=D_{n}(N)$. 
Proof. We may assume that $M$ has a cyclic and separating trace vector $\xi_{0}$. It is known in the theory of standard forms (see [6, Lemma 1.1]) that $P_{n}=$ $\overline{c o}\left\{\left[x_{i}^{*} x_{j} \xi_{0}\right] \in M_{n}(H) \mid x_{i} \in M\right\}$ is a self-dual cone in a Hilbert space $M_{n}(H)$; that is, the dual cone $P_{n}^{\prime}=\left\{\xi \in M_{n}(H) \mid(\xi, \eta) \geq 0\right.$ for all $\left.\eta \in P_{n}\right\}$ of $P_{n}$ coincides with itself. Put $Q_{n}=\overline{\operatorname{co}}\left\{\left[a_{i}^{*} c^{*} c a_{j} \xi_{0}\right] \mid a_{i} \in N, c \in M\right\}$ and $R_{n}=$ $\left\{\left[\xi_{i, j}\right] \in M_{n}(H) \mid \sum_{i, j=1}^{n} a_{i} J a_{j} J \xi_{i, j} \in P\right.$ for every $\left.a_{1}, \ldots, a_{n} \in N\right\}$, where $J$ denotes an isomorphic involution and $P$ a self-dual cone in $H$ related to $\xi_{0}$. We then have $Q_{n} \subset P_{n} \subset R_{n}, Q_{n} \subset Q_{n}^{\prime}$, and $Q_{n}^{\prime}=R_{n}$. One easily sees that $Q_{n}=P_{n}$ implies $R_{n}=P_{n}$. Conversely, $R_{n}=P_{n}$ implies $Q_{n}=P_{n}$ by using the Hahn-Banach Theorem. Hence we have the desired result by virtue of the following facts: For $x_{i, j} \in M$ and $a_{i} \in M$, we have

$$
\sum_{i, j=1}^{n} a_{i} J a_{j} J x_{i, j} \xi_{0}=\sum_{i, j=1}^{n} a_{i} x_{i, j} a_{j}^{*} \xi_{0} .
$$

If $\left[x_{i, j} \xi_{0}\right]$ belongs to $P_{n}$ for $x_{i, j} \in M$, then for $y_{i} \in M^{\prime}$

$$
\left(\left[x_{i, j}\right]\left[y_{i} \xi_{0}\right],\left[y_{i} \xi_{0}\right]\right)=\left(\left[x_{i, j} \xi_{0}\right],\left[y_{i}^{*} y_{j} \xi_{0}\right]\right) \geq 0
$$

because $\overline{c o}\left\{\left[y_{i}^{*} y_{j} \xi_{0}\right] \mid y_{i} \in M^{\prime}\right\}$ is a dual cone of $P_{n}$, which means $\left[x_{i, j}\right] \geq 0$.

Lemma 5. Let $A$ be an abelian von Neumann algebra.

(i) If $M$ is a von Neumann algebra and $N$ is a von Neumann subalgebra of $M$ such that $M_{n}(M)^{+}=C_{n}(N)$ for some $n \in \mathbb{N}$, then $M_{n}(M \otimes A)^{+}=C_{n}(N \otimes I)$.

(ii) If $M$ is a von Neumann algebra on a separable Hilbert space $H$ and $N$ is a von Neumann subalgebra of $M$ such that $M_{n}(M)^{+}=D_{n}(N)$ for some $n \in \mathbb{N}$, then $M_{n}(M \otimes A)^{+}=D_{n}(N \otimes I)$.

Proof. (i) We identify $M_{n}(M \otimes A)=(M \otimes A) \otimes M_{n}$ with $\left(M \otimes M_{n}\right) \otimes A$. Since $A$ is abelian, the positive cone $\left(\left(M \otimes M_{n}\right) \otimes A\right)^{+}$coincides with the strongly closed convex hull of the elements $x \otimes y$ for $x \in\left(M \otimes M_{n}\right)^{+}$and $y \in A^{+}$.

In fact, suppose that there exists an element $z_{0}$ in $\left(\left(M \otimes M_{n}\right) \otimes A\right)^{+}$which does not belong to the cone $\overline{c o}^{\sigma-w}\left\{x \otimes y \mid x \in\left(M \otimes M_{n}\right)^{+}, y \in A^{+}\right\}$. By the Hahn-Banach Theorem, there exists a $\sigma$-weakly continuous linear functional $\phi$ on $\left(M \otimes M_{n}\right) \otimes A$ such that $\phi\left(z_{0}\right)<0$ and $\phi(z) \geq 0$, where $z$ is any element of the above convex hull. However, $\phi$ must be a positive functional on $\left(M \otimes M_{n}\right) \otimes A$ by [7, Theorem 3.4], which is a contradiction. By the Kaplansky density theorem, the set of all elements $x \otimes y$ for $x \in\left(M \otimes M_{n}\right)$ and $y \in A^{+}$ is strongly dense in $\left(\left(M \otimes M_{n}\right) \otimes A\right)^{+}$.

Thus we have $\left(\left(M \otimes M_{n}\right) \otimes A\right)^{+}={\overline{\operatorname{co}^{5}}}^{\mathrm{s}}\left\{\left[x_{i}^{*} x_{j}\right] \otimes a^{*} a \mid x_{i} \in M, a \in A\right\}$. By assumption, we have

$$
\begin{aligned}
M_{n}(M \otimes A)^{+} & =\overline{\operatorname{co}}^{\mathrm{s}}\left\{\left[y_{i}^{*} c^{*} c y_{j}\right] \otimes a^{*} a \mid y_{i} \in N, c \in M, a \in A\right\} \\
& \left.=\overline{\operatorname{co}}^{\mathrm{s}}\left\{\left[y_{i} \otimes 1\right)^{*}(c \otimes a)^{*}(c \otimes a)\left(y_{j} \otimes 1\right)\right] \mid y_{i} \in N, c \in M, a \in A\right\} .
\end{aligned}
$$

(ii) Take an element $\left[x_{i, j}\right]$ in $D_{n}(N \otimes I)$. By identifying $A$ with $L^{\infty}(\Gamma, \mu)$, where $\Gamma$ is a locally compact Hausdorff space and $\mu$ is a positive Radon measure in $\Gamma$, each $x_{i, j} \in M \otimes A$ is expressed as

$$
x_{i, j}=\int_{\Gamma}^{\oplus} x_{i, j}(\gamma) d \mu(\gamma),
$$


where $x_{i, j}(\gamma)$ is an element of $M$. We then have, for any $a_{i} \in N$,

$$
\int_{\Gamma}^{\oplus} \sum_{i, j=1}^{n} a_{i}^{*} x_{i, j}(\gamma) a_{j} d \mu(\gamma)=\sum_{i, j=1}^{n}\left(a_{i}^{*} \otimes 1\right) x_{i, j}\left(a_{j} \otimes 1\right) \geq 0 .
$$

Since $H$ is separable, we have $\sum_{i, j=1}^{n} a_{i}^{*} x_{i, j}(\gamma) a_{j} \geq 0$ locally $\mu$-almost everywhere by [8, Proposition IV.7.12]. Since $N$ is separable in the strong topology, the above inequality holds for all $a_{i} \in N$. By assumption, we have $x_{i, j}(\gamma) \geq 0$ locally $\mu$-almost everywhere. It follows that

$$
\begin{aligned}
\left(\left[x_{i, j}\right]\left[\xi_{i}\right],\left[\xi_{i}\right]\right) & =\sum_{i, j=1}^{n}\left(x_{i, j} \xi_{j}, \xi_{i}\right) \\
& =\int_{\Gamma} \sum_{i, j=1}^{n}\left(x_{i, j}(\gamma) \xi_{j}(\gamma), \xi_{i}(\gamma)\right) d \mu(\gamma) \geq 0,
\end{aligned}
$$

for any $\left[\xi_{i}\right] \in\left(H \otimes L^{2}(\Gamma, \mu)\right) \otimes H_{n}$, of which each component is expressed as

$$
\xi_{i}=\int_{\Gamma}^{\oplus} \xi_{i}(\gamma) d \mu(\gamma), \quad \xi_{i}(\gamma) \in H .
$$

Therefore, $\left[x_{i, j}\right] \geq 0$. This completes the proof.

We shall next consider, in the matrix algebra $M$, the condition for $N$ that the cone $D_{n}(N)$ includes properly $M_{n}(M)^{+}$.

Lemma 6. If $k<m$ then $M_{n}\left(M_{k m}\right)^{+} \neq D_{n}\left(M_{k} \otimes I_{m}\right)$ for $n \geq 2$.

Proof. We first consider the case $n=2$. Suppose that $\left[x_{i, j}\right]\left(x_{i, j} \in M_{k m}=\right.$ $\left.M_{k} \otimes M_{m}, i, j=1,2\right)$ belongs to $D_{2}\left(M_{k} \otimes I_{m}\right)$ and $k<m$. We then have, for $a_{i} \in M_{k}$ and $\xi \in H_{k m}$,

$$
\begin{gathered}
\left(\left[\begin{array}{ll}
x_{1,1} & x_{1,2} \\
x_{2,1} & x_{2,2}
\end{array}\right]\left[\begin{array}{l}
\left(a_{1} \otimes 1_{m}\right) \xi \\
\left(a_{2} \otimes 1_{m}\right) \xi
\end{array}\right],\left[\begin{array}{l}
\left(a_{1} \otimes 1_{m}\right) \xi \\
\left(a_{2} \otimes 1_{m}\right) \xi
\end{array}\right]\right) \\
\quad=\left(\sum_{i, j=1}^{2}\left(a_{i}^{*} \otimes 1_{m}\right) x_{i, j}\left(a_{j} \otimes 1_{m}\right) \xi, \xi\right) \geq 0 .
\end{gathered}
$$

Put $a_{i}=\left[\alpha_{p, q}^{(i)}\right] \in M_{k}$ for $i=1,2$ and

$$
\boldsymbol{\xi}=\left[\begin{array}{c}
\xi_{1} \\
\vdots \\
\xi_{m}
\end{array}\right], \quad \xi_{j}=\left[\begin{array}{c}
\lambda_{1}^{(j)} \\
\vdots \\
\lambda_{m}^{(j)}
\end{array}\right],
$$

for $\alpha_{p, q}^{(i)} \in \mathbb{C}$ and $\lambda_{r}^{(j)} \in \mathbb{C}$. We then obtain the following equality:

$$
\begin{aligned}
\left(a_{i} \otimes 1_{m}\right) \xi & =\left[\begin{array}{lll}
a_{i} & & 0 \\
& \ddots & \\
0 & & a_{i}
\end{array}\right]\left[\begin{array}{c}
\xi_{1} \\
\vdots \\
\xi_{m}
\end{array}\right] \\
& =\sum_{q=1}^{k}{ }^{t}\left[\alpha_{1, q}^{(i)} \lambda_{q}^{(1)}, \ldots, \alpha_{k, q}^{(i)} \lambda_{q}^{(1)}, \ldots, \alpha_{1, q}^{(i)} \lambda_{q}^{(m)}, \ldots, \alpha_{k, q}^{(i)} \lambda_{q}^{(m)}\right]
\end{aligned}
$$


for $i=1,2$. Put

$$
\eta_{q}={ }^{t}\left[\alpha_{1, q}^{(1)}, \alpha_{2, q}^{(1)}, \ldots, \alpha_{k, q}^{(1)}, \alpha_{1, q}^{(2)}, \alpha_{2, q}^{(2)}, \ldots, \alpha_{k, q}^{(2)}\right] \in H_{2 k}
$$

and

$$
\zeta_{q}={ }^{t}\left[\lambda_{q}^{(1)}, \lambda_{q}^{(2)}, \ldots, \lambda_{q}^{(m)}\right] \in H_{m},
$$

for $q=1,2, \ldots, k$. If we consider that $\left[x_{i, j}\right]$ acts on $H_{2 k} \otimes H_{m}$, we get the inequality $\omega\left(\eta_{1} \otimes \zeta_{1}+\eta_{2} \otimes \zeta_{2}+\cdots+\eta_{k} \otimes \zeta_{k}, \eta_{1} \otimes \zeta_{1}+\eta_{2} \otimes \zeta_{2}+\cdots+\eta_{k} \otimes \zeta_{k}\right)\left(\left[x_{i, j}\right]\right) \geq$ 0 , where $\omega_{\xi, \eta}$ is denoted by $\omega(\xi, \eta)$. Conversely, if an element $\left[x_{i, j}\right]$ of $M_{2}\left(M_{k m}\right)$ satisfies the above inequality for every $\eta_{q} \in H_{2 k}$ and $\zeta_{q}=H_{m}$ $(q=1,2, \ldots, k),\left[x_{i, j}\right]$ belongs to $D_{2}\left(M_{k} \otimes I_{m}\right)$.

Now, suppose that any element of $D_{2}\left(M_{k} \otimes I_{m}\right)$ is positive. By identifying $M_{2 k m}$ with $M_{2 k} \otimes M_{m}$, the order of $M_{2 k} \otimes M_{m}$ is determined by the family of positive elements of order $k$ in $\left(M_{2 k} \otimes M_{m}\right)^{*}$.

In fact, we have

$$
\begin{aligned}
\omega\left(\eta_{1} \otimes \zeta_{1}+\cdots+\eta_{k} \otimes \zeta_{k}, \eta_{1} \otimes \zeta_{1}+\cdots+\eta_{k} \otimes \zeta_{k}\right) \\
=\sum_{s, t=1}^{k} \omega\left(\eta_{s}, \eta_{t}\right) \otimes \omega\left(\zeta_{s}, \zeta_{t}\right) .
\end{aligned}
$$

If $c_{i}$ is an arbitrary element of $M_{2 k}$ for $i=1,2, \ldots, k$, then we have

$$
\sum_{s, t=1}^{k} \omega\left(\eta_{t}, \eta_{s}\right)\left(c_{s}^{*} c_{t}\right)=\left(\sum_{s=1}^{k} c_{s} \eta_{s}, \sum_{s=1}^{k} c_{s} \eta_{s}\right) \geq 0 .
$$

Hence $\left[\omega\left(\eta_{t}, \eta_{s}\right)\right]$ is a positive element of $M_{k}\left(M_{2 k}\right)^{*}$. Similarly, $\left[\omega\left(\zeta_{t}, \zeta_{s}\right)\right]$ is a positive element of $M_{k}\left(M_{m}\right)^{*}$.

By [9, Theorem 2.2], $M_{2 k}$ or $M_{m}$ is $k$-homogeneous, which is a contradiction. We obtained the required results.

We next consider the case $n \geq 3$. By the above argument there exists an element $y=\left[y_{i, j}\right] \in D_{2}\left(M_{k} \otimes I_{m}\right)$ which does not belong to $M_{2}\left(M_{k m}\right)^{+}$. Put $Y=\left[Y_{i, j}\right]=\left[\begin{array}{ll}y & 0 \\ 0 & E\end{array}\right]$, where $Y_{i, j} \in M_{k m}$ and $E=I_{k m} \oplus \cdots \oplus I_{k m} \in M_{n-2}\left(M_{k m}\right)$. If $a_{i} \in M_{k} \otimes I_{m}$ for $i=1,2, \ldots, n$, then we have

$$
\sum_{i, j=1}^{n} a_{i}^{*} Y_{i, j} a_{j}=\sum_{i, j=1}^{2} a_{i}^{*} y_{i, j} a_{j}+\left(\sum_{i=3}^{n} a_{i}\right)^{*}\left(\sum_{i=3}^{n} a_{i}\right) \geq 0 .
$$

But $Y$ is not a positive matrix. This completes the proof.

We now obtain the following two theorems by using the above discussion, in which we investigate the factorization of positive cones of order $n$ of a finite von Neumann algebra of type I and an injective factor.

Theorem 7. Let $M$ be a finite von Neumann algebra of type I expressed as $M=\sum_{i=1}^{m \oplus} M_{k(i)} \otimes A_{k(i)}$ with $A_{k(i)}$ abelian von Neumann algebras. Put $N=$ $\sum_{i=1}^{m \oplus} N_{k(i)} \otimes I_{k(i)}$ with $N_{k(i)}$ von Neumann subalgebras of $M_{k(i)}$. Then, for each natural number $n \geq 2$, the following conditions are equivalent:

(i) $M_{n}(M)^{+}=C_{n}(N)$.

(ii) $M_{n}(M)^{+}=D_{n}(N)$.

(iii) Every $N_{k(i)}$ has a cyclic vector. 
Proof. (i) $\Rightarrow$ (ii) We may only consider the case $M=M_{k} \otimes A$. Since $A$ is abelian, $M_{n}(M)^{+}$coincides with the strongly closed convex hull of all elements $\left[x_{i, j}\right] \otimes a$ with $\left[x_{i, j}\right] \in M_{n}\left(M_{k}^{+}\right)$and $a \in A^{+}$by the proof of Lemma 5 . On the other hand, we have

$$
\begin{aligned}
C_{n}\left(N_{k} \otimes I\right) & =\overline{\operatorname{co}}^{\mathrm{s}}\left\{\left[\left(x_{i} \otimes 1\right)^{*} c^{*} c\left(x_{j} \otimes 1\right)\right] \mid x_{i} \in N_{k}, \quad c \in M_{k} \otimes A\right\} \\
& =\overline{c 0}^{\mathrm{s}}\left\{\left[x_{i}^{*} y^{*} y x_{i}\right] \otimes a \mid x_{i} \in N_{k}, y \in M_{k}, \quad a \in A^{+}\right\} .
\end{aligned}
$$

By assumption, we get the equality $M_{n}\left(M_{k}\right)^{+}=C_{n}\left(N_{k}\right)$. By Lemmas 4 and 5 , we have $M_{n}\left(M_{k} \otimes A_{k}\right)^{+}=D_{n}\left(N_{k} \otimes I\right)$.

(ii) $\Rightarrow$ (iii) Let $N$ be a von Neumann subalgebra of a matrix algebra $M_{k}$ and $N$ have no cyclic vector. It then suffices to prove $M_{n}\left(M_{k}\right)^{+} \neq D_{n}(N)$ for $n \geq 2$. We may consider $N$ as $M_{m} \otimes I_{m^{\prime}} \oplus B\left(\subset M_{k}\right)$ with $m<m^{\prime}$, where $B$ is a von Neumann algebra on $H_{k-m m^{\prime}}$. By Lemma 6 we can find an element $\left[x_{i, j}\right]$ in $M_{n}\left(M_{m} \otimes M_{m^{\prime}}\right)$ such that $\left[x_{i, j}\right] \in D_{n}\left(M_{m} \otimes I_{m^{\prime}}\right)$ and $\left[x_{i, j}\right]$ is not positive. Put $X_{i, j}=x_{i, j} \oplus \delta_{i j} 1_{B} \in M_{k}$. If $a_{i} \in M_{m m^{\prime}}$ and $b_{i} \in B$ for $i=1,2, \ldots, n$, then

$$
\sum_{i, j=1}^{n}\left(a_{i} \oplus b_{i}\right)^{*} X_{i, j}\left(a_{j} \oplus b_{j}\right)=\sum_{i, j=1}^{n} a_{i}^{*} x_{i, j} a_{j} \oplus \sum_{i=1}^{n} b_{i}^{*} b_{i} .
$$

Then $\left[X_{i, j}\right]$ belongs to $D_{n}(N)$, but it is not positive.

(iii) $\Rightarrow$ (i) We apply Proposition 2 and Lemmas 4 and 5.

Theorem 8. Let $H$ be a separable Hilbert space.

(i) If $M$ is an injective von Neumann algebra on $H$, then there exists an abelian *-subalgebra $A$ of $M$ such that $M_{n}(M)^{+}=C_{n}(A)$ for every $n \in \mathbb{N}$.

(ii) If $M$ is an injective factor (or semifinite, injective von Neumann algebra), then there exists an abelian *-subalgebra $A$ of $M$ such that $M_{n}(M)^{+}=D_{n}(A)$ for every $n \in \mathbb{N}$.

Proof. The statement (i) is due to [4, Proposition 2.2].

(ii) Suppose that $M$ is an injective factor on a separable Hilbert space $H$. $M$ is then a Krieger's factor (cf. [2, Theorems 7.5, 7.7; 3, Corollary 2.4]). By [1, Theorem 1], there exists an increasing sequence $\left\{\varepsilon_{k}\right\}$ of normal conditional expectations of $M$ onto finite-dimensional subalgebras $N_{k}$ with $M=\left(\bigcup_{k+1}^{\infty} N_{k}\right)^{-s}$. Then, for every $x \in M, \varepsilon_{k}(x)$ converges strongly to $x$ by [1, Lemma 2]. Fix a natural number $k$. Since $N_{k}$ is decomposed into a finite direct sum of matrix algebras, we construct a maximal abelian *-subalgebra $A_{k}$ of $N_{k}$, each component of which consists of all diagonal matrices. We then have $\left(M_{n}\left(N_{k}\right)^{+}=D_{n}\left(A_{k}\right)\right.$ by Proposition 2. Since all maximal abelian *-subalgebras of a finite-dimensional von Neumann algebra are spatially isomorphic, there exists a maximal abelian *-subalgebra $A_{k+1}$ of $N_{k+1}$ such that $A_{k} \subset A_{k+1}$ and $M_{n}\left(N_{k+1}\right)^{+}=D_{n}\left(A_{k+1}\right)$. By induction, we put $A=\bigcup_{k=1}^{\infty} A_{k}$, which is an abelian *-subalgebra of $M$. Take an element $\left[x_{i, j}\right]$ in $D_{n}(A)$. We then have, for each $k$,

$$
\sum_{i, j=1}^{n} a_{i}^{*} \varepsilon_{k}\left(x_{i, j}\right) a_{j}=\varepsilon_{k}\left(\sum_{i, j=1}^{n} a_{i}^{*} x_{i, j} a_{j}\right) \geq 0,
$$

for all $a_{i} \in A_{k}$. Hence $\left[\varepsilon_{k}\left(x_{i, j}\right)\right] \geq 0$. As $k$ tends to $\infty$, we obtain $\left[x_{i, j}\right] \geq 0$. 
Suppose now that $M$ is a semifinite, injective von Neumann algebra. We may only consider that $M$ is continuous. By using the reduction theory, $M$ is written as

$$
M=R_{0} \otimes B_{0} \oplus R_{0} \otimes B_{1} \otimes B(K),
$$

where $R_{0}$ is a hyperfinite factor of type $\mathrm{II}_{1}$ and $B_{0}$ and $B_{1}$ are abelian von Neumann algebras because of the unicity of $R_{0}$. By the first half of the proof, we can find abelian von Neumann subalgebras $E$ and $F$ of $R_{0}$ and $B(K)$ with $M_{n}\left(R_{0}\right)^{+}=D_{n}(E)$ and $M_{n}(B(K))^{+}=D_{n}(F)$, respectively. Put $A=$ $E \otimes I \oplus E \otimes I \otimes F$. We then have, by Lemma $5, M_{n}(M)^{+}=D_{n}(A)$. This completes the proof.

We note here that a maximal abelian von Neumann subalgebra on $H$ having a cyclic vector is maximal abelian in $B(H)$ (see, for example, [5, Proposition 2.8.3]). The above theorem shows that there exists a *-subalgebra $N$ of an injective factor $M$ of type II or III satisfying the conditions (i) and (ii) and having no cyclic vector.

Finally, we shall state the following remark for a completely positive map. Recall that a positive linear map $\phi$ of a von Neumann algebra $M_{1}$ to $M_{2}$ is said to be completely positive if, for every natural number $n$, a multiplicity map $\phi \otimes 1_{n}$ maps a positive cone in $M_{n}\left(M_{1}\right)$ into a positive cone in $M_{n}\left(M_{2}\right)$.

Remark 9. Let $M_{1}$ and $M_{2}$ be von Neumann algebras and $N_{1}$ and $N_{2}$ subalgebras of $M_{1}$ and $M_{2}$ satisfying, for all natural numbers $n, C_{n}\left(N_{1}\right)=M_{n}\left(M_{1}\right)^{+}$ and $D_{n}\left(N_{2}\right)=M_{n}\left(M_{2}\right)^{+}$, respectively. Suppose that $\phi$ is a normal positive map of $M_{1}$ into $M_{2}$. Then $\phi$ is completely positive if and only if $\phi$ satisfies, for all natural numbers $n$, the relation

$$
\sum_{i, j=1}^{n} b_{i}^{*} \phi\left(a_{i}^{*} c^{*} c a_{j}\right) b_{j} \geq 0,
$$

for every $a_{1}, \ldots, a_{n} \in N_{1}, c \in M_{1}$, and $b_{1}, \ldots, b_{n} \in N_{2}$.

\section{ACKNOWLEDGMENT}

The author thanks the members of the seminar in Sendai, especially Professors T. Okayasu, H. Takemoto, and S. Yamagami, for their useful suggestions.

\section{REFERENCES}

1. A. Connes, On hyperfinite factors of type III and Krieger's factors, J. Funct. Anal. 18 (1975), 318-327.

2. $\ldots$, Classification of injective factors, Cases $\mathrm{II}_{1}, \mathrm{II}_{\infty}, \mathrm{III}_{\lambda}, \lambda \neq 1$, Ann. of Math. (2) 104 (1976), 73-115.

3. U. Haagerup, Connes' bicentralizer problem and uniqueness of the injective factor of type $\mathrm{III}_{1}$, Acta Math. 158 (1987), 95-147.

4. Y. Miura, A certain factorization of selfdual cones associated with standard forms of injective factors, Tokyo J. Math. 13 (1990), 73-86.

5. G. K. Pedersen, $C^{*}$-algebras and their automorphism groups, Academic Press, London, New York, and San Francisco, 1979.

6. L. M. Schmitt and G. Wittstock, Characterization of matrix-ordered standard forms of $W^{*}$-algebras, Math. Scand. 51 (1982), 241-260. 
7. T. Takasaki and J. Tomiyama, Stinespring type theorems for various types of completely positive maps associated to operator algebras, Math. Japon. 27 (1982), 129-139.

8. M. Takesaki, Theory of operator algebras. I, Springer-Verlag, New York, Heidelberg, and Berlin, 1979.

9. J. Tomiyama, On the difference of $n$-positivity and complete positivity in $C^{*}$-algebras, J. Funct. Anal. 49 (1982), 1-9.

Department of Mathematics, College of Humanities and Social Sciences, Iwate UniVERSITY, MORIOKA 020, JAPAN 